\title{
Statistical Investigation of Current-Voltage Characterization in Single Molecule-Metal Junctions
}

\author{
T. Kirchner, B. Briechle, E. Scheer \\ Fachbereich Physik, Universität Konstanz, D-78457 Konstanz, Germany \\ J. Wolf, T. HuhN \\ Fachbereich Chemie, Universität Konstanz, D-78457 Konstanz, Germany \\ AND A. ERBE* \\ Helmholtz-Zentrum Dresden-Rossendorf, D-01328 Dresden, Germany
}

\begin{abstract}
We show statistical measurements of single molecule-metal contacts using the mechanically controllable break junction technique. The measurements are carried out in a solvent, in order to allow in situ binding of the molecules to the metallic contacts during the measurements. Statistics is gathered by opening and closing the junctions repeatedly and recording current-voltage characteristics at various stages of the opening and closing curves. By modeling the data with a single level model we can extract parameters such as the position of the molecular energy level, which carries the current, and the coupling between the metal and the molecule. In first experiments we use this method to characterize different anchoring groups, which mediate the mechanical and electrical coupling between the metallic electrodes and the molecules. We use tolane molecules, which are structurally simple, as model systems for this purpose.
\end{abstract}

PACS: 73.23.-b, 73.63.Rt, 81.07.Nb

\section{Introduction}

The central topic of molecular electronics is the development of stable structures, which can conduct electrical charges in a controlled way. Ideally, such a structure is given by a single molecule that is connected to two macroscopic, conducting leads. Experimentally, this approach turns out to be difficult to realize. A large number of contacting techniques has been investigated in order to find a device structure, which provides a reliable, mechanically stable contact with a large conductance [1-3] (for simplicity we call these contacts stable and transparent). Mechanically controllable break junctions (MCBJ) have been used for producing contacts to molecular structures since the very early years of molecular electronics [4]. The technique could be improved in later years until it was shown that single molecule-metal contacts could be produced in vacuum at room temperature [5-7] and at low temperature [8]. The main drawback of this technique is given by the fact that the molecule-metal contact is formed ex situ, by applying a droplet of the molecular solution before opening the MCBJ. The formation of a molecular junction therefore relies on the migration of the molecules towards the center of the molecular wire and the binding of the molecules at the tips of the wires. The exact structure of the molecules in vacuum is generally not known, because all standard characterization tools for molecular structures work on molecules in a solvent or in self-assembled monolayers (SAMs) [9]. We thus de-

* corresponding author; e-mail: a.erbe@hzdr.de veloped a technique to characterize the molecules in a solvent.

Here we report characterization of short organic tolane molecules, which are attached to the gold electrodes via different anchoring groups. In order to verify that the observed characteristics do not stem from artefacts arising from metallic nanocontacts in solution, we first characterize the behavior of purely metallic contacts. Comparison with contacts, which are opened and closed in a molecular solution, shows clearly the influence of the molecules. The results of single current-voltage curves ( $I-V$ curves) are compared to a single level model, which allows us to extract the coupling of the molecules to the electrodes and the position of the energy level, which contributes to the current [10].

\section{Experimental methods}

The strength of the MCBJ technique in liquid environments is its potential to study a large number of molecular junctions by repeatedly opening and closing a metallic junction in a molecular solvent. The presence of the molecules in this solvent provides fresh molecules, once a new metallic contact is opened. This allows us to gather a large number of data on a specific molecular species.

The measurement setup is shown in Fig. 1. The MCBJ is defined on a flexible substrate and placed in a mechanism that enables bending the substrate in a very controlled way. At the center of the substrate we defined a small, free-standing gold bridge (100 nm wide and $2 \mu \mathrm{m}$ long) using electron beam lithography and dry etching. Upon bending of the substrate the bridge is opened 


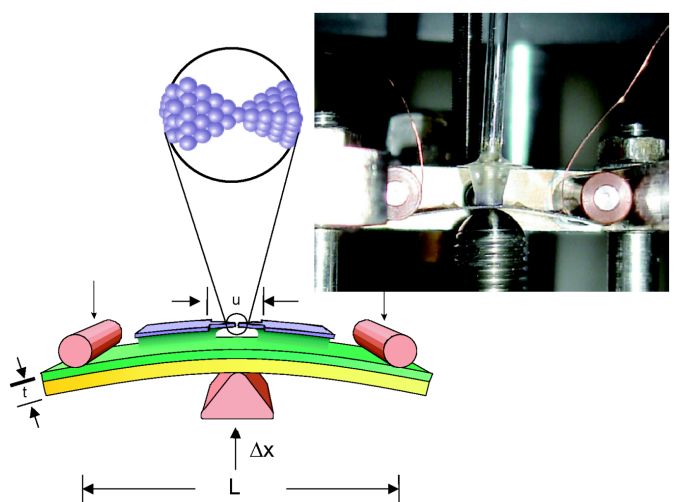

Fig. 1. Scheme of the MCBJ setup. Upper right: image of the pipette pressing onto the metallic junction.

slowly and a single atom contact is formed [11]. Further opening leads to a tunneling contact between two atomically sharp tips. A pipette is filled with a molecular solution (see Table for concentration) or a pure solvent and positioned at the center of the substrate on top of the lithographically defined metallic constriction. The substrate is then bent, and the gold bridge is opened in the solution.

\section{TABLE}

Concentration of measured molecules.

\begin{tabular}{c|c|c}
\hline \hline Molecule & Concentration $[\mathrm{mmol} / \mathrm{l}]$ & Solvent \\
\hline thiol & 1.0 & toluol \\
cyano & 1.6 & toluol \\
nitril & 0.014 & toluol
\end{tabular}

\subsection{Pure solvents}

The first characterization of the electrode samples is performed in the pure solvent, in order to exclude artefacts coming from transport through the solvent. We record the conductance as a function of the position of the pushing rod, which is bending the sample. The current between two metallic electrodes decays exponentially with increasing distance in a nonpolar solvent [12]. Thus, the behavior of tunneling contacts in vacuum and in solvent are qualitatively similar. The shape of $I-V$ curves measured in a solvent, however, shows some deviation from the behavior which is known from vacuum conditions. At very low currents (below $10 \mathrm{pA}$ ) we observe hysteretic behavior which is most likely caused by electrochemical effects. Since the current level in molecular junctions is 5 to 6 orders of magnitude larger, measurements on the molecules are not perturbed by this contribution.

\subsection{Characterization of molecules}

Opening and closing curves in a molecular solvent show steps in the regime of $G \ll G_{0}$, typically around
$G=10^{-3}$ to $10^{-4} G_{0}$ for the tolane molecules characterized in our experiments. Measurements of $I-V$ curves in this conductance range result in a curve shape differing clearly from the hysteretic behavior observed for pure solvent. We can group the $I-V$ curves into three sets, depending on the conductance range in which they are measured and on the shape of the $I-V$ curve. At high conductance $\left(G>10^{-2} G_{0}\right)$ we observe a linear behavior, which is caused by direct tunneling between the two metallic electrodes. At intermediate values of the conductance $\left(10^{-3} G_{0}>G>10^{-5} G_{0}\right)$ a large part of the $I-V$ curves shows nonlinear behavior.

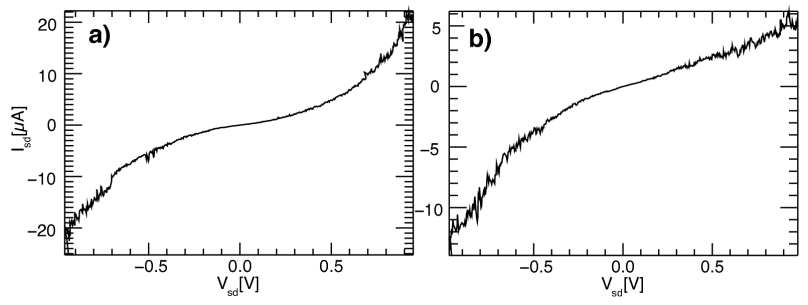

Fig. 2. $\quad I-V$ curves measured on BNT. (a) Symmetric $I-V$ curve, (b) asymmetric $I-V$ curve.

A typical example of such curves is shown in Fig. 2. For some of these curves the absolute value of the current is symmetric with respect to $V_{\mathrm{sd}}=0$ (as shown in Fig. 2), while others show asymmetries of various orders of magnitude. In order to explain the shape of the $I-V$ curves, which has been observed in a larger number of experiments on molecular structures to date, we use a model that takes the contribution of a single molecular energy level to the current through the junction into account. This model will be described in the following section. At very low conductance values $\left(G<1 \times 10^{-5} G_{0}\right)$ we again observe tunneling with a linear shape of the $I-V$ curves, but at much lower currents.

\section{Single level model}

We assume that only one molecular orbital takes part in the electronic conduction through the molecular junction. This orbital can be either the highest occupied molecular orbital (HOMO) or the lowest unoccupied molecular orbital (LUMO). The orbital, which lies in energy closest to the Fermi energy of gold $\left(E_{\mathrm{F}}\right)$ conducts electrons through the molecule. The relative position of the energy of this orbital with respect to the Fermi energy of the metal $\left(E_{0}\right)$ and the coupling of the molecular state to the electrodes, $\Gamma_{\mathrm{L}, \mathrm{R}}$, determine the magnitude of the current. When the molecules are brought into contact with the electrodes, the strength of the coupling between molecule and metal causes a shift of the molecular energy and a broadening of the molecular energy level. Thus both the value of $E_{0}$ and the width of this orbital depend on $\Gamma$. The current through the molecule can be separated in a current to the left electrode $I_{\mathrm{L}}$ and a current to the right electrode $I_{\mathrm{R}}$. They are given by 


$$
I_{\mathrm{L}}=\frac{e \Gamma_{\mathrm{L}}}{\hbar}\left[2 f_{\mathrm{L}}\left(E_{0}\right)-N_{0}\right]
$$

and

$$
I_{\mathrm{R}}=\frac{e \Gamma_{\mathrm{R}}}{\hbar}\left[N_{0}-2 f_{\mathrm{R}}\left(E_{0}\right)\right] .
$$

Here, $N_{0}$ is the occupation number of the molecular state out of equilibrium, and $f_{\mathrm{L}, \mathrm{R}}$ are the Fermi functions at the left and right electrode, respectively. A stationary current requires $I_{\mathrm{L}}=I_{\mathrm{R}}$, thus we find

$$
I=I_{\mathrm{L}}=I_{\mathrm{R}}=\frac{2 e}{\hbar} \frac{\Gamma_{\mathrm{L}} \Gamma_{\mathrm{R}}}{\Gamma_{\mathrm{L}}+\Gamma_{\mathrm{R}}}\left[f_{\mathrm{L}}\left(E_{0}\right)-f_{\mathrm{R}}\left(E_{0}\right)\right] .
$$

Coupling of the molecular level to the metallic leads causes a broadening of the level which leads, according to Breit and Wigner [13], to a density of states given by

$$
D(E)=\frac{2}{\pi} \frac{\Gamma_{\mathrm{L}} \Gamma_{\mathrm{R}}}{\left(E-E_{0}\right)^{2}+\left(\Gamma_{\mathrm{L}}+\Gamma_{\mathrm{R}}\right)^{2}},
$$

and Eq. (3) is changed to

$$
\begin{aligned}
I & =\frac{2 e}{h} \int \frac{4 \Gamma_{\mathrm{L}} \Gamma_{\mathrm{R}}}{\left(E-E_{0}\right)^{2}+\left(\Gamma_{\mathrm{L}}+\Gamma_{\mathrm{R}}\right)^{2}} \\
& \times\left[f_{\mathrm{L}}(E)-f_{\mathrm{R}}(E)\right] \mathrm{d}(E) .
\end{aligned}
$$

The difference in the Fermi energies $f_{\mathrm{L}}-f_{\mathrm{R}}$, which is caused by application of a source-drain bias $V_{\text {sd }}$ across the junction, drives the current through the molecule. Thus, we can evaluate Eq. (5) for different values of $V_{\text {sd }}$ and obtain an $I-V$ curve. The result is shown in Fig. 3 for different values of $\Gamma$ and $E_{0}$.

For the symmetric case, $\Gamma=\Gamma_{\mathrm{L}}=\Gamma_{\mathrm{R}}$, the $I-V$ curves have a nonlinear shape, which resembles closely the shape shown in Fig. 2a. The amplitude of the current depends on both the values of $\Gamma$ and of $E_{0}$, as can be seen in Fig. 3a and b, respectively. An increasing value of $\Gamma$ leads to an increase in the coupling between metal and molecule and in the broadening of the energy level. Thus the current value rises and the nonlinearity of the $I-V$ curve becomes less pronounced towards larger values of $\Gamma$. A decrease in the value of $E_{0}$ is equivalent to a closer alignment of the molecular energy level and the Fermi energy of the contacting metal $E_{\mathrm{F}}$. As long as $E_{0} \ll e V_{\max } / 2$, where $V_{\max }$ is the maximum source drain bias, which can be applied to the junction without destroying it, the shape of the $I-V$ curve does not change and the current rises with decreasing $E_{0}$.

Once the energy supplied by the source-drain bias and $E_{0}$ are on a comparable scale, the junction approaches resonant tunneling. Close to the resonance condition the conductance rises and decreases when the voltage is increased further. This maximum in conductance leads to a sharp increase of the current as a function of voltage before the resonance condition and to an almost constant value of the current at higher voltages. In Fig. 3b $I-V$ curves for various values of $E_{0}$ are shown, $E_{0}$ decreases from red to blue. It can be seen that for large values of $E_{0}$ the nonlinear behavior of our measured molecules is reproduced, while smaller values show

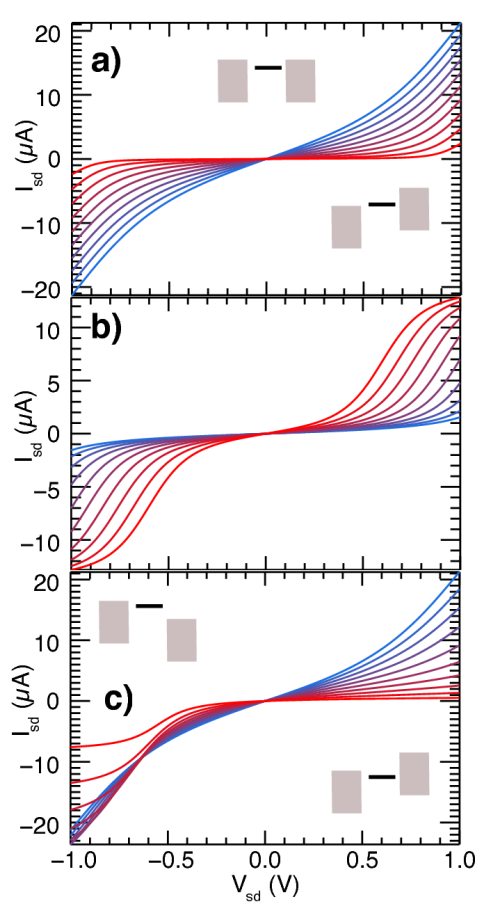

Fig. 3. $I-V$ curves calculated for different values of $\Gamma$ and $E_{0}$ in the single level model. (a) Variation of $\Gamma$ in a symmetric junction. The position of the molecular level was fixed at $E_{0}=0.5 \mathrm{eV}, 0.01 \mathrm{eV} \leq \Gamma \leq$ $0.1 \mathrm{eV}$. (b) Variation of $E_{0}$ in a symmetric junction. $\Gamma=0.03 \mathrm{eV}, 0.3 \mathrm{eV} \leq \mathrm{E}_{0} \leq 0.7 \mathrm{eV}$. (c) Variation of $\Gamma_{1}$ in an asymmetric junction. $\Gamma_{2}=0.1 \mathrm{eV}, E_{0}=0.5$, $0.01 \mathrm{eV} \leq \Gamma_{1} \leq 0.1 \mathrm{eV}$.

a step-like behavior. From this we can conclude that we observe off-resonant tunneling through a single molecular energy level.

Experimentally, we find a large number of asymmetric curves, in which one polarity of the voltage leads to a larger conductance than the other, as shown in Fig. $2 \mathrm{~b}$. In order to explain such results we need to assume that the coupling to the two metallic electrodes is different, i.e. $\Gamma_{\mathrm{L}} \neq \Gamma_{\mathrm{R}}$. In this scenario, the voltage drop across the linkers will be different on the two sides, as well. Due to this coupling the molecular level will follow the electrode, to which it is coupled, stronger. The insets in Fig. 3c indicate this situation. Thus, $E_{0}$ is no longer independent of the voltage but given in the simplest assumption by

$$
E_{0}(V)=E_{0}+\frac{\Gamma_{\mathrm{L}}-\Gamma_{\mathrm{R}}}{\Gamma_{\mathrm{L}}+\Gamma_{\mathrm{R}}} \frac{e V}{2} .
$$

This leads to a diode-like behavior, as shown in Fig. 3c. In our measurements we find a large number of such asymmetric curves, an example is shown in Fig. 2b. Fits to the single level work well for this set of measurements.

\section{Choice of molecules}

It is clear that the coupling between molecules and metallic electrodes depends on the molecular anchoring 
group, which mediates the mechanical and electrical stability of the molecule inside the junction. We therefore measured molecules, which consist of an identical, simple central structure and are equipped with various anchoring groups. The structures of the molecules are shown in Fig. 4. The basic molecule is 1,2-bisphenylethene (tolane) (BTT). BTT uses the commonly used thiol anchoring group. We use this molecule as a benchmark for the measurements in the other anchoring groups, because a large number of successful experiments have been reported based on molecules, which link to gold electrodes using this special chemistry $[3,5,14]$. On the other hand, nitrogen is known to react with gold surfaces. This interaction can lead to a strong binding of molecules to surfaces, if there are free electrons available at the junction. This scenario has been confirmed using scanning tunneling microscope (STM) breakjunctions and amino terminated molecules [15].

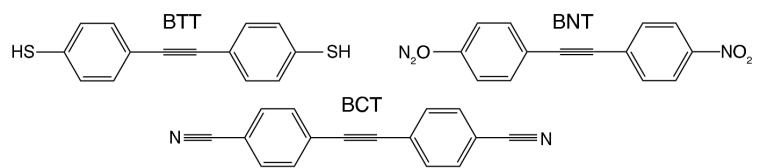

Fig. 4. Characterized molecules: BTT: 4,4'-bisthiotolane, BNT: 4,4'-bisnitrotolane, BCT: $4,4^{\prime}$-biscyanotolane.

In our measurements, we compare a nitro group, in which the nitrogen is coordinated to two oxygen atoms (BNT), and a cyano group, where the nitrogen is connected via a triple bond to the neighboring $\mathrm{C}$-atom (BCT). We expect good coupling for the nitro group, but rather poor coupling for the cyano groups.

\section{Experimental results}

Figure 5 shows typical results for measurements of $I-V$ curves on BTT in a conductance range, in which plateaus in the opening and closing curves (see Sect. 2.2) are observed. This set of $I-V$ curves is characteristic for measurements on all three measured molecular species. It can be seen that experimental results for a single $I-V$ curve vary by a large degree even for the same molecule. Therefore, we cannot draw any meaningful conclusion from a single $I-V$ curve and use statistical evaluation of a large number of measured junctions in order to analyze differences between the molecules. For this purpose we classify all measured $I-V$ curves into the following categories:

Symmetric. These $I-V$ curves resemble the curve shape, which is shown in Fig. 2a and can be modelled using the single level model with symmetric coupling.

Asymmetric. These $I-V$ curves resemble the curve shape, which is shown in Fig. $2 \mathrm{~b}$ and can be modelled using the single level model with asymmetric coupling.

Linear. These $I-V$ curves show a constant conductance. The most probable reason for this shape is direct tunneling between the metallic contacts or direct metallic contact.

Steps. Some curves show a stepwise increase of the current, which cannot easily be explained by molecular properties.

Kinks. In some measurements sudden rearrangements of the metallic contacts cause a stepwise increase or decrease of the conductance.

Jumps. These $I-V$ curves are too difficult to categorize because the junctions were too unstable.

Of course, the transitions between the categories are in some cases arbitrary, but a first characterization can be given by looking at the statistical distribution of the curves, as shown in Fig. 6.

From this preliminary categorization we can already see that the majority of junctions measured on BTT shows symmetric $s$-shape behavior, while the majority for BCT is found to show jumps. For BNT about $30 \%$ of all junctions show either symmetric or asymmetric behavior and about $30 \%$ show jumps. This shows that the thiol anchoring group provides the most stable connection between metal and molecule, while the cyano group provides rather poor stability. Based on results known from literature and our own expectations as discussed in Sect. 4, this is the expected behavior.

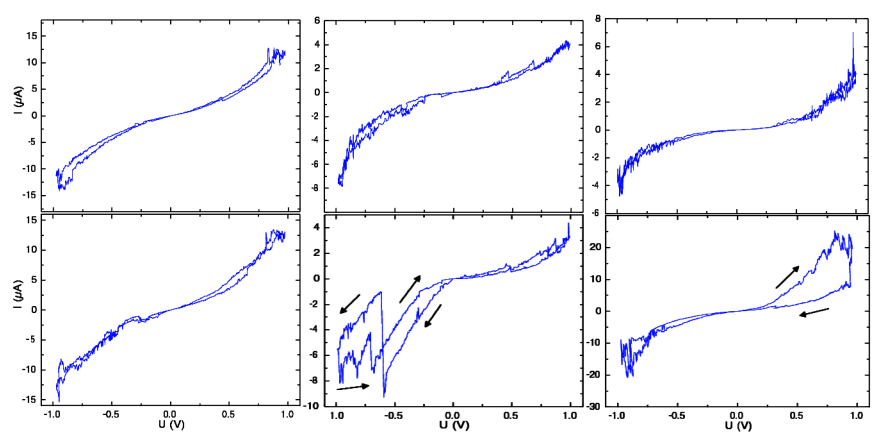

Fig. 5. Selection of $I-V$ curves measured on BTT.

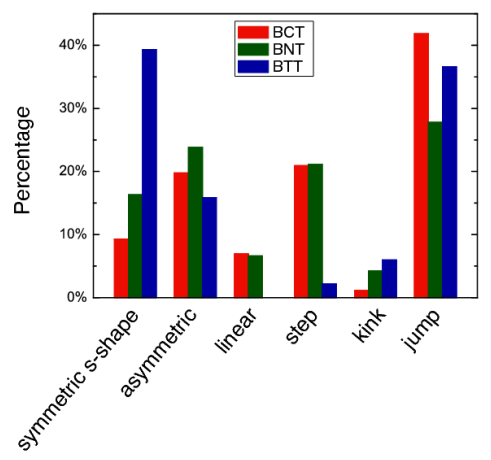

Fig. 6. Statistical distribution of curve shapes for all measured molecules.

The situation for the nitro group is more complex. The large number of asymmetric curves gives some hint that 
the mechanical coupling for the nitro group is less reliable than for the thiol group. This then leads to a different coupling on the two sides of the molecule and, as explained in Sect. 3, to asymmetric curves.

We investigate the $I-V$ curves in more detail in order to understand details of the coupling between the metal and the molecule. This is done by analyzing the symmetric $I-V$ curves only, because in this scenario the single level model can be used using two independent parameters only to describe the measured curves. We extract from all symmetric $s$-shaped curves the values for $\Gamma$ and $E_{0}$ and compare these values. In order to understand the role of the anchoring group on the conductance of the molecular junctions, we calculate the transmission $T$ of each junction, which is given according to Landauer's formula $\left(G=\left.\frac{2 e^{2}}{h} T(E, V)\right|_{V=0}\right)$ and Eq. (5) as

$$
\left.T(E, V)\right|_{V=0}=\frac{4 \Gamma_{\mathrm{L}} \Gamma_{\mathrm{R}}}{\left[E-E_{0}\right]^{2}+\left[\Gamma_{\mathrm{L}}+\Gamma_{\mathrm{R}}\right]^{2}} .
$$

The transmission values for BTT range from $T=0.01$ to $T=0.13$, for BNT from $T=0.14$ to $T=0.34$ and for BCT from $T=2 \times 10^{-7}$ to $T=4 \times 10^{-5}$. This wide range of transmission values shows that the influence of the various anchoring groups can be resolved by statistical evaluation of the parameters, which determine the transmission. We therefore plot $E_{0}$ and $\Gamma$ as a function of $T$ for the three molecules. The result is shown in Fig. 7 . The position of the molecular orbital with respect to the Fermi energy of the metals does not show a dependence on the transmission for all three molecular species. The coupling, however, shows a linear tendency for BTT and $\mathrm{BCT}$, while there is no apparent dependence for BNT.

\section{Discussion}

Judging from the relative number of symmetric $I-V$ curves, we can conclude that the mechanical stability of the molecule-metal bond is indeed best for the thiol anchoring group. This can be expected from known results on SAM formation [9] and earlier studies in monomolecular junctions. The connection between the nitro group and the metallic electrodes is less reliable mechanically, as we conclude from the large number of asymmetric curves. The mechanically least stable connection is the cyano group. Here the triple bond of the last nitrogen atom withdraws electron density from the connection and a stable connection is therefore not formed. This can be seen from the very low number of $s$-shaped $I-V$ curves, which we find for this molecule, and the comparably large number of unstable junctions.

Apart from the mechanical stability the junctions need to show an electrical connection with a large transmission, if the anchoring groups should be considered as suitable linkers for molecular electronics. Judging from the design of the molecules we can expect that the well conjugated backbone of the tolane molecules acts as a molecular wire [16]. Therefore, a low overall transmission of the system is expected to be caused by the interplay of the
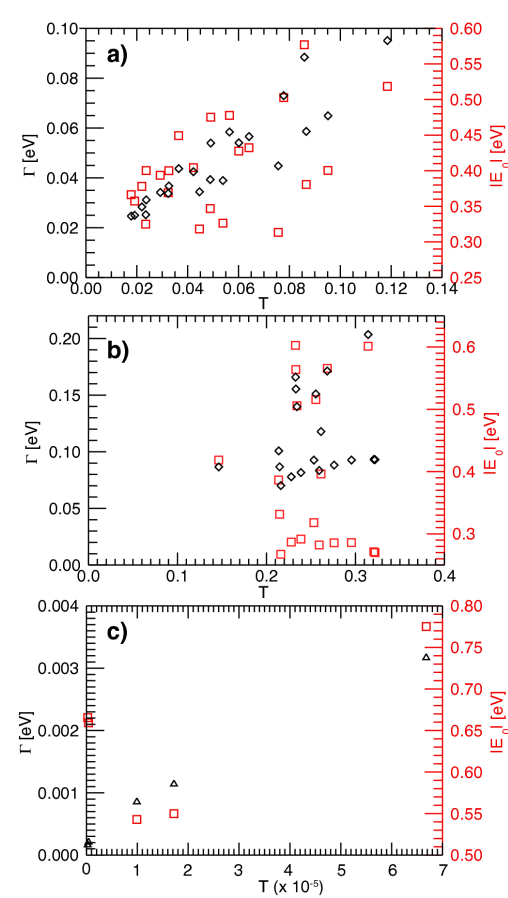

Fig. 7. Energy of the molecular level $E_{0}$ and the coupling between leads and molecule $\Gamma$ as a function of the transmission of the system $T$ for three different anchoring groups: (a) thiol, (b) nitro, and (c) cyano.

molecule with the metallic electrodes via the anchoring groups.

In our experiments, we find the highest transmission for the nitro group, followed by the thiol group; the transmission of the cyano group is by far the lowest. The position of the molecular orbital in the case of the BCT varies between $E_{0}=0.5 \mathrm{eV}$ and $E_{0}=0.8 \mathrm{eV}$, while it lies in between $E_{0}=0.3 \mathrm{eV}$ and $E_{0}=0.6 \mathrm{eV}$ for BTT and BNT. The value of $E_{0}$ is determined by the energetic position of the molecular orbital and by the charge transfer between metal and molecule. For BCT, the offset between the energies in the connected state is larger than for the other two molecular species. This is caused by the weak coupling of the anchoring group $(0.1 \mathrm{meV}<\Gamma<4 \mathrm{meV})$, which impedes electron transfer between metal and molecules. Thus, in the case of the cyano group, the poor mechanical stability and the low transmission of the junction lead to molecular junction with poor electric characteristics. The transmission in case of BTT is larger and the offset between molecular orbital and the Fermi energy of the metal is smaller. The coupling $\Gamma$ is between 20 and $100 \mathrm{meV}$ allowing for a more effective charge transfer between metal and molecule. We find a tendency that $\Gamma$ increases linearly with the transmission $T$. From this behavior we can judge that a single molecular level transports current between the metallic electrodes. The exact value of the coupling $\Gamma$ and the transmission $T$ depends on geometrical details. Junctions with BNT molecules show an even larger transmis- 
sion than BTT junctions. When looking at the dependence of $\Gamma$ on the transmission $T$, however, we find no tendency as was the case for both BTT and BCT. This indicates that the conductance in BNT is more complicated than for the other two molecular species. A comparison with first principles transport calculations shows that most likely conductance in the BNT junctions occurs through more than one single molecular orbital [10]. Thus, the evaluation scheme as outlined above is not valid for the BNT molecules.

\section{Conclusion}

We have shown that, using the MCBJ technique, we can characterize the conductance behavior of single organic molecules in a liquid environment. We have tested tolane molecules with various anchoring groups. We see clear differences in the behavior of these anchoring groups, which we can characterize by comparing the measured $I-V$ curves with a single level model. From this comparison we understand that molecules, which are linked to gold electrodes via thiol anchoring groups, form mechanically stable junctions, which allow transport through single molecular orbitals with a high transmission. Thus, for the case of the molecules under study here, thiol anchoring groups appear to be the ideal linkers for further use in developing molecular electronics.

\section{References}

[1] A. Erbe, S. Verleger, Acta Phys. Pol. A 115, 455 (2009).

[2] H.B. Akkerman, B. de Boer, J. Phys., Condens. Matter 20, 13001 (2008).

[3] H.S.J. van der Zant, Y.-V. Kervennic, M. Poot, K. O'Neill, Z. de Groot, J, M. Thijssen, H, B. Heersche, N. Stuhr-Hansen, T. Bjornholm, D. Vanmaekelbergh, C.A. van Walree, L.W. Jenneskens, Faraday Discuss. 131, 347 (2006).
[4] M.A. Reed, C. Zhou, C.J. Muller, T.P. Burgin, J.M. Tour, Science 278, 252 (1997).

[5] J. Reichert, R. Ochs, D. Beckmann, H.B. Weber, M. Mayor, H. v. Löhneysen, Phys. Rev. Lett. $\mathbf{8 8}$, 176804 (2002).

[6] M. Mayor, C.v. Hahnisch, H.B. Weber, J. Reichert, D. Beckmann, Angew. Chem. Int. Ed. 41, 1183 (2002).

[7] E. Lörtscher, J.W. Ciszek, J. Tour, H. Riel, Small 2, 973 (2006).

[8] J. Reichert, H.B. Weber, M. Mayor, H. v. Löhneysen, Appl. Phys. Lett. 82, 4137 (2003).

[9] B. de Boer, H. Meng, D.F. Perepichka, J. Zheng, M.M. Frank, Y.J. Chabal, Z. Bao, Langmuir 2003, 4272 (2003).

[10] L.A. Zotti, Th. Kirchner, J.-C. Cuevas, F. Pauly, Th. Huhn, E. Scheer, A. Erbe, Small 6, 1529 (2010).

[11] J.M. van Ruitenbeek, A. Alvarez, I. Pineyro, C. Grahmann, P. Joyez, M.H. Devoret, D. Esteve, C. Urbina, Rev. Sci. Instrum. 67, 108 (1996).

[12] L. Gruter, M.T. Gonzalez, R. Huber, M. Calame, C. Schonenberger, Small 1, 1067 (2005).

[13] G. Breit, E. Wigner, Phys. Rev. 49, 519 (1936).

[14] M.A. Reed, C. Zhou, C.J. Muller, T.P. Burgin, J.M. Tour, Science 278, 252 (1997).

[15] S.Y. Quek, L. Venkataraman, H.J. Choi, S.G. Louie, M.S. Hybertsen, J.B. Neaton, Nano Lett. 7, 3477 (2007).

[16] W.A. Reinerth, L. Jones, II, T.P. Burgin, C.-W. Zhou, C.J. Muller, M.R. Deshpande, M.A. Reed, J.M. Tour, Nanotechnology 9, 246 (1998). 